\title{
Trajectories of E-Cigarette and Conventional Cigarette Use Among Youth
}

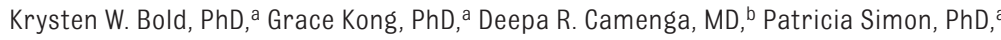
Dana A. Cavallo, PhD, ${ }^{a}$ Meghan E. Morean, PhD, ${ }^{,}$Suchitra Krishnan-Sarin, PhDa

BACKGROUND: Electronic cigarette (e-cigarette) use is common among youth, and there are concerns that e-cigarette use leads to future conventional cigarette use. We examined longitudinal associations between past-month cigarette and e-cigarette use to characterize the stability and directionality of these tobacco use trajectories over time.

METHODS: High school students ( $N=808,53 \%$ female) completed surveys across 3 waves (2013, 2014, and 2015) in 3 public schools in Connecticut. Using autoregressive crosslagged models, we examined bidirectional relationships between past-month cigarette and e-cigarette use over time. Models were adjusted for covariates related to tobacco use (ie, sex, race/ethnicity, socioeconomic status, and use of other tobacco products).

RESULTS: Past-month e-cigarette use predicted future cigarette use (wave 1-2: odds ratio $[\mathrm{OR}]=7.08,95 \%$ confidence interval $[\mathrm{CI}]=2.34-21.42$; wave $2-3: \mathrm{OR}=3.87,95 \% \mathrm{CI}=$ 1.86-8.06). However, past-month cigarette use did not predict future e-cigarette use (wave $1-2: \mathrm{OR}=2.02,95 \% \mathrm{CI}=0.67-6.08$; wave $2-3: \mathrm{OR}=1.90,95 \% \mathrm{CI}=0.77-4.71$ ). Additionally, frequency of cigarette and e-cigarette use increased over time. By wave $3,26 \%$ of cigarette users and $20.5 \%$ of e-cigarette users reported using 21-30 days out of the past month.

conclusions: E-cigarette use was associated with future cigarette use across 3 longitudinal waves, yet cigarette use was not associated with future e-cigarette use. Future research needs to examine mechanisms through which e-cigarette use leads to cigarette use. E-cigarette regulation and prevention programs may help prevent future use of cigarettes among youth.

Departments of ${ }^{a}$ Psychiatry and ${ }^{b}$ Emergency Medicine, School of Medicine, Yale University, New Haven, Connecticut; and ${ }^{C}$ Department of Psychology, Oberlin College, Oberlin, Ohio

Dr Bold contributed to the conceptualization of the study, developed and tested the hypotheses reported in the manuscript, ran all statistical analyses, and wrote the primary manuscript draft; Drs Kong, Camenga, Simon, Cavallo, and Morean contributed to the conceptualization of the study and the development of the self-report survey and critically reviewed drafts of the manuscript; Dr Krishnan-Sarin secured study funding, led the conceptualization of the study and the development of the self-report survey, and critically reviewed drafts of the manuscript; and all authors approved the final manuscript as submitted.

Dol: https://doi.org/10.1542/peds.2017-1832

Accepted for publication Sep 28, 2017

Address correspondence to Krysten W. Bold, PhD, Department of Psychiatry, Yale University School of Medicine, 34 Park St, CMHC-SAC, New Haven, CT 06519. E-mail: krysten.bold@yale.edu

PEDIATRICS (ISSN Numbers: Print, 0031-4005; Online, 1098-4275).

Copyright @ 2018 by the American Academy of Pediatrics
WHAT'S KNOWN ON THIS SUBJECT: Electronic cigarette (e-cigarette) use rates are high among youth, and there are concerns that e-cigarette use confers risk for future conventional cigarette use. Prospective research is needed to characterize the stability and directionality of these tobacco use trajectories over time.

WHAT THIS STUDY ADDS: Past-month e-cigarette use predicted future conventional cigarette use across 3 longitudinal waves among high school youth. Cigarette use did not predict subsequent e-cigarette use. E-cigarette regulation and prevention programs are needed to reduce future use of conventional cigarettes among youth.
To cite: Bold KW, Kong G, Camenga DR, et al. Trajectories of E-Cigarette and Conventional Cigarette Use Among Youth. Pediatrics. 2018;141(1):e20171832 
Electronic cigarettes (e-cigarettes) are battery-operated devices used to vaporize liquid solutions that may contain nicotine, flavors, and other chemicals. E-cigarettes are now the most commonly used tobacco product among youth; an estimated 3 million US adolescents currently use e-cigarettes, ${ }^{1}$ including many who have never used conventional cigarettes. $^{2,3}$ There are concerns that e-cigarette use among youth may lead to conventional cigarette smoking, which is a leading cause of morbidity and mortality in the United States. ${ }^{4}$

Longitudinal cohort studies provide some of the strongest scientific evidence to assess the prospective relationship between e-cigarette use and future cigarette use. To date, results from several longitudinal studies indicate that e-cigarette use among nonsmoking youth increases the likelihood of future use of conventional cigarettes..$^{5-10}$ Specifically, the pooled odds ratio (OR) in a recent meta-analysis of studies of adolescents and young adults (aged 14-30) indicates that those who had ever used e-cigarettes were 3.62 times more likely to report using cigarettes at follow-up compared with those who had not used e-cigarettes. ${ }^{11}$ This finding was robust and remained significant when adjusting for known risk factors associated with cigarette smoking, including demographic, psychosocial, and behavioral variables such as cigarette susceptibility.

Thus, there is growing evidence to support the concern that e-cigarette use is associated with future cigarette use. However, previous studies have focused on the unidirectional relationship between 2 time points, examining the association between baseline e-cigarette use and future cigarette use but not the stability of these relationships over time or the potential reverse directionality. E-cigarette and cigarette use may be highly correlated over time such that the use of either product is associated with the use of the other, in which case cigarette use would also confer risk for future e-cigarette use.

To better understand the stability and directionality of these associations over time, we used a cross-lagged model to examine the direct and reciprocal relationships between e-cigarette and cigarette use across 3 waves $(2013,2014$, and 2015) using longitudinal survey evidence from high school students in Connecticut. With this analytic approach, we can simultaneously model cross-lagged effects (ie, relationships between e-cigarette use at 1 wave predicting cigarette use at a future wave and vice versa) while controlling for previous levels of the variables across waves (ie, direct effects of earlier use on future use of the same product), ${ }^{12}$ thereby ruling out the possibility that a cross-lagged effect is merely representing a strong correlation between the likelihood of e-cigarette and cigarette use at a given point in time. If the crosslagged relationship is unidirectional over multiple waves such that e-cigarette use predicts future cigarette use but not vice versa, this would further support the notion that e-cigarette use is a risk factor for future conventional cigarette smoking.

\section{METHODS}

Longitudinal data were collected in school-wide surveys across 3 time points (wave 1: fall 2013; wave 2: spring 2014; and wave 3: spring 2015). Surveys were repeated across 3 Connecticut high schools that were selected across different district reference groups (ie, school groupings that vary on the basis of characteristics such as family income levels, parental education and occupation levels, and the use of a non-English language in the home ${ }^{13}$ to include diverse demographic and socioeconomic characteristics. Individual paper-and-pencil surveys were distributed during homeroom periods at each wave of the survey administration. Parents were contacted in advance of the study and could indicate if they did not want their child to participate. Students were informed that their participation was voluntary and that data were anonymous. Study procedures were approved by the Yale University Institutional Review Board and school administrators.

It has been suggested that more accurate reports of youth substance use are obtained from anonymous surveys, so all surveys were anonymous to encourage honest responding. ${ }^{4,15}$ Unique 5 -factor identification codes (eg, day value from date of birth, school, sex) were used to match student responses longitudinally by following previously validated procedures. ${ }^{16,17}$ Out of 1408 students, $n=1098$ were matched from wave 1 to 2 , and $n=972$ were matched from wave 2 to 3 . These match rates are comparable to those observed in other regional and national longitudinal surveys that used procedures for either anonymous ${ }^{16}$ or identifiable survey matching. 5,6 Those who were matched across all 3 waves $(n=808)$ did not significantly differ from those who were not matched longitudinally in terms of sociodemographic characteristics (eg, sex, race) or substance use (eg, ever or past-month use of cigarettes or e-cigarettes), and comparable match rates were observed across schools and grades, reducing potential concerns about attrition bias.

\section{Baseline Covariates}

In Table 1, we present sample characteristics, including sociodemographic variables and baseline use of other tobacco products. 
TABLE 1 Baseline Characteristics for the Matched Longitudinal Sample $(N=808)$

\begin{tabular}{|c|c|}
\hline Variable & Value \\
\hline \multicolumn{2}{|l|}{ Sex, No. (\%) } \\
\hline Male & $380(47.0)$ \\
\hline Female & $428(53.0)$ \\
\hline Age $^{a}$, mean (SD) & $15.04(0.90)$ \\
\hline $\mathrm{SES}^{\mathrm{b}}$, mean (SD) & $5.92(1.38)$ \\
\hline \multicolumn{2}{|l|}{ Race/ethnicityc, No. (\%) } \\
\hline White & $708(87.6)$ \\
\hline Asian & $46(5.7)$ \\
\hline Hispanic and/or Latino & $41(5.1)$ \\
\hline Black or African American & $21(2.6)$ \\
\hline American Indian or Alaskan Native & $8(1.0)$ \\
\hline Native Hawaiian or Pacific Islander & $6(0.7)$ \\
\hline Middle Eastern & $7(0.9)$ \\
\hline Other & $3(0.4)$ \\
\hline \multicolumn{2}{|c|}{ Ever use of other tobacco products ${ }^{d}$, No. (\%) } \\
\hline Yes & $157(19.4)$ \\
\hline No & $651(80.6)$ \\
\hline
\end{tabular}

a Age data available for $n=769$; range: $13-17$ y old.

${ }^{b}$ SES was assessed by using the Family Affluence Scale (Boyce et $\mathrm{al}^{18}$ ), data were available for $n=795$, and there was a possible range of 0 (low) to 8 (high).

${ }^{c}$ Race/ethnicity values add up to $>100 \%$ because students could select $>1$ category

d Ever use of other tobacco products (ie, cigars, hookah, blunts, smokeless tobacco)

\section{Demographics}

Students reported demographic characteristics including sex (male or female), age, and race/ethnicity (white, black or African American, Hispanic and/or Latino, Asian, American Indian or Alaskan Native, Native Hawaiian or other Pacific Islander, Middle Eastern, or other). Socioeconomic status (SES) was assessed by using the Family Affluence Scale, which has been shown to be a reliable and valid measure of SES among adolescents. ${ }^{18}$ The 4 -item Family Affluence Scale assessed the following: (1) whether an adolescent's family owns a car, van, or truck (no = 0 , yes = 1); (2) whether an adolescent has his or her own bedroom (no $=0$, yes = 1); (3) the number of laptops and/or computers an adolescent's family owns (none $=0,1=1,2=2,>2=$ 3 ); and (4) whether an adolescent's family had vacationed in the past 12 months (not at all $=0$, once $=1$, twice $=$ 2 , more than twice $=3$ ). Responses were added to create a total SES score.

\section{Ever Use of Other Tobacco Products}

Ever use of other tobacco products (ie, cigars, hookah, blunts, smokeless tobacco) was assessed by asking students if they had ever tried each product (yes or no) at wave 1.

\section{Longitudinal Outcome Measures \\ E-Cigarette Use} each wave by asking, "Have you ever tried an e-cigarette?" (yes or no) and, "How many days out of the past 30 days did you use e-cigarettes?" (open-ended response, 0-30).

\section{Cigarette Use}

Cigarette use was measured at each wave by asking, "Have you ever tried a cigarette, even just 1 or 2 puffs?" (yes or no) and, "During the past 30 days, on how many days did you smoke a cigarette (even just 1 or 2 puffs)?" Categorical response options included the following: "none," "1 day," "2 days," "3 to 5 days," "6 to 10 days," "11 to 20 days," "21 to 28 days," and "everyday."

\section{Primary Outcomes}

To capture recent use, which may be less prone to recall bias, the primary outcomes of interest were past-month use ( 1 = yes, $0=$ no) of cigarettes and e-cigarettes at each wave. If data on past-month use were
E-cigarette use was measured at missing and youth reported never trying the product in their lifetime, past-month use was imputed as $0=$ no use ( $n=10$ records, $0.4 \%$ of cases).

\section{Data Analysis}

Analyses were run by using Mplus (version 7.4). Outcome data were missing for $6.5 \%$ of cases across waves and were handled by using maximum likelihood estimation with robust standard errors. Pastmonth cigarette and e-cigarette use outcomes were modeled as binary variables with a logit link function. School was included as a covariate across all waves to account for potential school cohort effects. Path models were used to simultaneously estimate the following: (1) the autoregressive direct effects within cigarette and e-cigarette use across waves (ie, the association between e-cigarette and cigarette use at each wave and use of the same product at the next wave) and (2) the reciprocal predictive pathways between e-cigarette and cigarette use at each wave to use of the other product at the next wave (eg, e-cigarette use at wave 1 predicting cigarette use at wave 2 and vice versa) (see Fig 1).

Baseline variables such as sociodemographic characteristics and use of other tobacco products (ie, cigars, hookah, blunts, smokeless tobacco) were selected a priori as covariates on the basis of previous literature. ${ }^{19,20}$ Results were consistent across the models in which the direct and reciprocal effects were estimated without including any covariates $(n=808)$ and the model in which the effects were estimated including all baseline covariates (ie, sex, race/ethnicity, SES, use of other tobacco products; $n=795$ ). Therefore, results are presented from adjusted models including all covariates.

Lastly, frequencies of e-cigarette and cigarette product use were examined at each wave (Table 2). The 


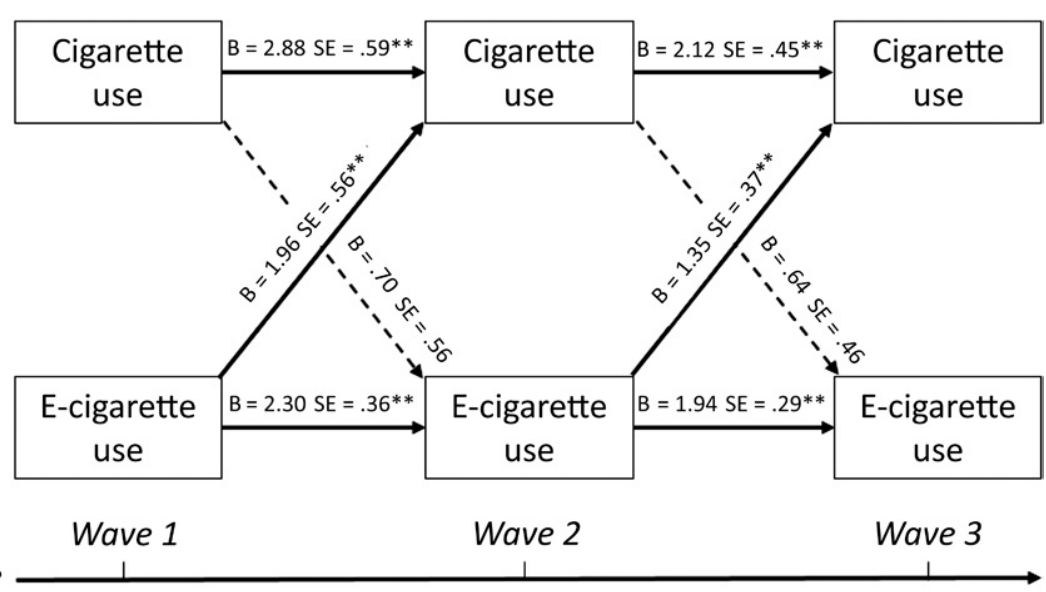

Time

\section{FIGURE 1}

Relationships between past-month cigarette and e-cigarette use among youth across 3 longitudinal waves $(N=808)$. Values represent unexponentiated path estimates $(B)$ and SEs. Path estimates can be exponentiated to obtain ORs. Past-month use is coded as yes or no. Estimates are adjusted for covariates (ie, sex, race, SES, other tobacco use, and school). Significant paths are denoted in a solid line $(* \star P<.01)$ and nonsignificant paths are denoted in a dashed line.

TABLE 2 Descriptive Statistics of Past 30-Day Cigarette and E-Cigarette Use by Wave $(N=808)$

\begin{tabular}{lccc}
\hline Variable & Wave 1: 2013 & Wave 2: 2014 & Wave 3: 2015 \\
\hline Age, mean (SD) & $15.0(0.9)$ & $15.5(1.0)$ & $16.4(1.0)$ \\
Past-month cigarette use, No. (\%) & $39(4.8)^{\mathrm{a}}$ & $44(5.4)^{\mathrm{a}}$ & $69(8.5)^{\mathrm{b}}$ \\
Past-month e-cigarette use, No. (\%) & $72(8.9)^{\mathrm{a}}$ & $97(12.0)^{\mathrm{b}}$ & $117(14.5)^{\mathrm{b}}$ \\
Days of cigarette use, No. (\%) & & & \\
$1-5 \mathrm{~d}$ & $29(3.6)^{\mathrm{a}}$ & $27(3.3)^{\mathrm{a}}$ & $38(4.7)^{\mathrm{a}}$ \\
$6-10 \mathrm{~d}$ & $2(0.2)^{\mathrm{a}}$ & $6(0.7)^{\mathrm{a}}$ & $8(1.0)^{\mathrm{a}}$ \\
$11-20 \mathrm{~d}$ & $4(0.5)^{\mathrm{a}}$ & $7(0.9)^{\mathrm{a}}$ & $5(0.6)^{\mathrm{a}}$ \\
$21-30 \mathrm{~d}$ & $4(0.5)^{\mathrm{a}}$ & $4(0.5)^{\mathrm{a}}$ & $18(2.2)^{\mathrm{b}}$ \\
Days of e-cigarette use, ${ }^{\mathrm{c}}$ No. (\%) & & \\
$1-5 \mathrm{~d}$ & $42(5.2)^{\mathrm{a}}$ & $60(7.4)^{\mathrm{a}, \mathrm{b}}$ & $69(8.5)^{\mathrm{b}}$ \\
$6-10 \mathrm{~d}$ & $16(2.0)^{\mathrm{a}}$ & $14(1.7)^{\mathrm{a}}$ & $11(1.4)^{\mathrm{a}}$ \\
$11-20 \mathrm{~d}$ & $3(0.4)^{\mathrm{a}}$ & $12(1.5)^{\mathrm{b}}$ & $13(1.6)^{\mathrm{b}}$ \\
$21-30 \mathrm{~d}$ & $11(1.4)^{\mathrm{a}}$ & $11(1.4)^{\mathrm{a}}$ & $24(3.0)^{\mathrm{b}}$ \\
\hline
\end{tabular}

Frequency values are expressed as the number (and percent) of the total matched longitudinal sample $(N=808)$. a,b superscripts denote column proportions that differ significantly from one another $(p<.05)$ calculated using a chi-square test. ${ }^{\circ}$ Number of days of e-cigarette use was recoded into numerical categories to match categorical responses of cigarette use frequency.

number of days of e-cigarette use was recoded into numerical categories to match categorical responses of cigarette use frequency. $\chi^{2}$ analyses were used to explore changes in frequencies over time.

\section{RESULTS}

Results from the path models (Fig 1) indicated significant autoregressive direct effects (ie, stability in use over time). As expected, past-month use of e-cigarettes and cigarettes at wave 1 predicted greater odds of pastmonth use of that same product at wave 2 (cigarette: OR $=17.74,95 \%$ confidence interval $[\mathrm{CI}]=5.56-56.56$; e-cigarette: $\mathrm{OR}=9.95,95 \% \mathrm{CI}=4.92$ 20.13). Similarly, past-month use of e-cigarettes and cigarettes at wave 2 predicted greater odds of past-month use of that same product at wave 3 (cigarette: $\mathrm{OR}=8.30,95 \% \mathrm{CI}=3.43-$ 20.10; e-cigarette: $\mathrm{OR}=7.00,95 \%$ $\mathrm{CI}=3.96-12.40$ ).

Additionally, our results indicated that there were significant reciprocal pathways between past-month e-cigarette use at each wave and future cigarette use. Specifically, individuals using e-cigarettes in the past month at wave 1 were $>7$ times more likely to report subsequent cigarette use at wave 2 (OR $=7.08,95 \% \mathrm{CI}=2.34-21.42$ ) when compared with those not using e-cigarettes. Furthermore, those using e-cigarettes in the past month at wave 2 were close to 4 times more likely to report subsequent cigarette use at wave $3(\mathrm{OR}=3.87$, $95 \% \mathrm{CI}=1.86-8.06$ ) when compared with those not using e-cigarettes. Conversely, past-month cigarette use at each wave was not significantly predictive of future e-cigarette use (wave 1-2: $\mathrm{OR}=2.02,95 \% \mathrm{CI}=0.67$ 6.08; wave $2-3: \mathrm{OR}=1.90,95 \% \mathrm{CI}=$ $0.77-4.71$ ). Thus, the effects were unidirectional such that youth using e-cigarettes were at greater risk for future cigarette use compared with those not using e-cigarettes, whereas the reverse relationship (cigarette use predicting future e-cigarette use) was not observed.

To further characterize cigarette and e-cigarette use, rates of product use were examined over time (Table 2). Overall, the rates of pastmonth use of both cigarettes and e-cigarettes significantly increased in the longitudinal sample over time (cigarettes: $\chi^{2}[N=2424]$, $P=.001$; e-cigarettes: $\chi^{2}[N=2424]$, $P=.001)$, almost doubling from wave 1 (2013) to wave 3 (2015). Frequency of use (measured as the number of days of use in the past month) also increased significantly over time for both cigarettes $\left(\chi^{2}\right.$ [N=2424], $P=.003$ ) and e-cigarettes $\left(\chi^{2}[N=2424], P=.001\right)$. By wave 3 , a greater proportion of youth reported using cigarettes on most days in the past month (ie, 21-30 days) when compared with waves 1 or 2. Additionally, significantly higher rates of low (1-5 days), moderate (11-20 days), and heavy (21-30 days) e-cigarette use were observed in wave 3 compared with earlier waves. By $2015,26 \%$ of past-month 
cigarette users and $20.5 \%$ of pastmonth e-cigarette users reported heavy use, characterized by using on the majority of the days during the month (21-30 days), which is an increase from $10.3 \%$ (cigarette) and $15.3 \%$ (e-cigarette) of users in 2013.

\section{DISCUSSION}

This study is the first to examine reciprocal relationships between past-month e-cigarette and cigarette use among high school youth using 3 waves of matched longitudinal data. We found that e-cigarette use is prospectively associated with a greater risk of future conventional cigarette use, while controlling for the autoregressive effects of stability of use over time. Importantly, we also observed that this risk is unidirectional, such that cigarette use was not significantly associated with e-cigarette use over time. The observed relationships were consistent across unadjusted models and when including covariates known to relate to cigarette and e-cigarette use (eg, sociodemographic characteristics, other tobacco use). Furthermore, rates of pastmonth cigarette and e-cigarette use increased significantly across our 3 waves of assessment, indicating more youth were using these products over time, which is consistent with epidemiologic evidence of higher rates of tobacco use as youth age. ${ }^{1,21}$ The rising frequency of recent e-cigarette use among youth over time is concerning, especially in light of evidence that e-cigarette use is a significant risk factor for future conventional cigarette use.

In future studies, researchers should examine potential mediators to better understand possible mechanisms that account for the observed directionality of the association between e-cigarette use and future cigarette use. For example, adolescents may be more likely to use e-cigarettes before conventional cigarettes because of factors unique to e-cigarette products, such as perceptions that e-cigarettes are less harmful than conventional cigarettes, ${ }^{2,22}$ the widespread availability of unique e-cigarette liquid flavors that may be especially appealing to youth, ${ }^{23,24}$ and limited enforcement or restrictions on youth access to e-cigarettes (eg, through online sales). ${ }^{25,26}$ At the same time, there is evidence that the adolescent brain is highly sensitive to the rewarding effects of nicotine, ${ }^{27,28}$ so e-cigarette use may provide early exposure to the reinforcing pharmacological effects of nicotine, which may increase the likelihood of transitioning to conventional cigarettes. Furthermore, nicotine may be delivered more efficiently through cigarettes than e-cigarettes depending on the e-cigarette device, ${ }^{29,30}$ so youth who use conventional cigarettes may find e-cigarettes less reinforcing and be less likely to transition in the reverse direction: from cigarette to e-cigarette use over time. Given the observational nature of the current survey study, we are unable to ascertain the specific causal mechanisms accounting for the association between e-cigarette use and future cigarette use. Yet our findings suggest that early prevention and intervention efforts as well as policies targeting youth e-cigarette use may be needed to reduce future conventional cigarette use among youth. Prevention efforts may include new e-cigarette regulatory policies given that the deeming rule now extends the regulatory authority of the Food and Drug Administration to other tobacco products, including e-cigarettes. ${ }^{31}$ As e-cigarette regulatory policies are enforced and product regulations change, continued research will be needed to understand how these strategies influence youth e-cigarette and cigarette use behaviors.

The current study has several important strengths, including the use of matched longitudinal data from the same high school students across 3 time points $(2013,2014$, and 2015) and a cross-lagged model with which we assessed the stability and directionality of associations between e-cigarettes and cigarettes over time. Furthermore, we used anonymous survey procedures that are known to improve the validity of substance use reporting among youth. With the current results, we extend previous longitudinal research that is focused on ever use of cigarettes and e-cigarettes, which may primarily capture youth who experiment with the product a single time, by examining past-month rates and frequencies to better quantify recent product use over time.

Several limitations should also be noted. For example, the sample comprised high school students from Connecticut, with the majority identifying as white, so our results may not generalize to other geographic or more diverse sociodemographic areas. Additionally, there may be other product characteristics (eg, nicotine strength) or covariates (eg, advertisement exposure, parental tobacco use) not assessed in this study that may relate to cigarette and e-cigarette use over time that should be considered in future investigations. Although we controlled for use of other tobacco products at baseline in the current study, it will be important for researchers to also consider how use patterns change across multiple tobacco products longitudinally. In our study, we intended to focus on adolescents during high school given the unique vulnerability to tobacco product use during this developmental period, and further research is needed to characterize trajectories between e-cigarette 
and cigarette use among people of other ages, including persistence of use into adulthood. Lastly, we focused on binary outcomes of pastmonth use of the products given the small sample sizes across the full range of use frequency. However, our preliminary analyses indicated higher rates of e-cigarette and cigarette use over time, consistent with national samples. Future largescale longitudinal studies are needed to address the question of how frequency of use of 1 product relates to future use of another product.

\section{CONCLUSIONS}

This study is the first to examine reciprocal associations between cigarette and e-cigarette use among youth across 3 longitudinal waves. The observed relationship between cigarette and e-cigarette use over time was unidirectional. E-cigarette use was associated with future cigarette use across 3 longitudinal waves, yet cigarette use was not associated with future e-cigarette use. Potential mediators of this effect should be examined in future research to better understand the mechanisms through which e-cigarette use increases the risk for future cigarette use. Prevention and intervention efforts and policies targeting youth e-cigarette use may be needed to reduce future conventional tobacco use among youth.

\section{ABBREVIATIONS}

CI: confidence interval e-cigarette: electronic cigarette OR: odds ratio SES: socioeconomic status

FINANCIAL DISCLOSURE: The authors have indicated they have no financial relationships relevant to this article to disclose.

FUNDING: Supported in part by grants from the National Institute on Drug Abuse and the Food and Drug Administration Center for Tobacco Products (P50DA036151, P50DA009241, T32DA019426, and L40DA042454). The content is solely the responsibility of the authors and does not necessarily represent the official views of the National Institutes of Health or the Food and Drug Administration. Funded by the National Institutes of Health (NIH).

POTENTIAL CONFLICT OF INTEREST: The authors have indicated they have no potential conflicts of interest to disclose.

COMPANION PAPER: A companion to this article can be found online at www.pediatrics.org/cgi/doi/10.1542/peds.2017-2850.

\section{REFERENCES}

1. Singh T, Arrazola RA, Corey CG, et al. Tobacco use among middle and high school students-United States, 20112015. MMWR Morb Mortal Wkly Rep. 2016;65(14):361-367

2. US Department of Health and Human Services. E-Cigarette Use Among Youth and Young Adults: A Report of the Surgeon General. Atlanta, GA: US Department of Health and Human Services, Centers for Disease Control and Prevention, National Center for Chronic Disease Prevention and Health Promotion, Office on Smoking and Health; 2016

3. Johnston LD, 0'Malley PM, Miech RA, Bachman JG, Schulenberg JE. Monitoring the Future: National Results on Adolescent Drug Use. 2014 Overview: Key Findings on Adolescent Drug Use. Ann Arbor, MI: Institute for Social Research, the University of Michigan; 2015

4. US Department of Health and Human Services. The Health Consequences of Smoking - 50 Years of Progress: $A$ Report of the Surgeon General. Atlanta, GA: US Department of Health and Human Services, Centers for Disease
Control and Prevention, National Center for Chronic Disease Prevention and Health Promotion, Office on Smoking and Health; 2014:17

5. Miech R, Patrick ME, O’Malley PM, Johnston LD. E-cigarette use as a predictor of cigarette smoking: results from a 1-year follow-up of a national sample of 12th grade students [published online ahead of print February 6 , 2017]. Tob Control. 2017;10.1136/ tobaccocontrol-2016-053291

6. Primack BA, Soneji S, Stoolmiller M, Fine MJ, Sargent JD. Progression to traditional cigarette smoking after electronic cigarette use among US adolescents and young adults. JAMA Pediatr. 2015;169(11):1018-1023

7. Barrington-Trimis JL, Urman R, Berhane K, et al. E-cigarettes and future cigarette use. Pediatrics. 2016;138(1):e20160379

8. Leventhal AM, Strong DR, Kirkpatrick $M G$, et al. Association of electronic cigarette use with initiation of combustible tobacco product smoking in early adolescence. JAMA. 2015;314(7):700-707
9. Wills TA, Knight R, Sargent JD, Gibbons FX, Pagano I, Williams RJ. Longitudinal study of e-cigarette use and onset of cigarette smoking among high school students in Hawaii. Tob Control. 2017;26(1):34-39

10. Unger JB, Soto DW, Leventhal A. E-cigarette use and subsequent cigarette and marijuana use among Hispanic young adults. Drug Alcohol Depend. 2016;163:261-264

11. Soneji S, Barrington-Trimis JL, Wills TA, et al. Association between initial use of e-cigarettes and subsequent cigarette smoking among adolescents and young adults: a systematic review and meta-analysis. JAMA Pediatr. 2017;171(8):788-797

12. Selig JP, Little TD. Autoregressive and cross-lagged panel analysis for longitudinal data. In: Laursen B, Little TD, Card NA, eds. Handbook of Developmental Research Methods. New York, NY: Guilford Press; 2012:265-278

13. Prowda P; Connecticut State Department of Education. District reference groups, 2006. Available at: http://sdeportal.ct.gov/Cedar/Files/ 
Pdf/Reports/db_drg_06_2006.pdf. Accessed September 1, 2017

14. Murray DM, Perry CL. The measurement of substance use among adolescents: when is the 'bogus pipeline' method needed? Addict Behav. 1987;12(3):225-233

15. Bjarnason T, Adalbjarnardottir S. Anonymity and confidentiality in school surveys on alcohol, tobacco, and cannabis use. J Drug Issues. 2000;30(2):335-343

16. Grube JW, Morgan M, Kearney KA. Using self-generated identification codes to match questionnaires in panel studies of adolescent substance use. Addict Behav. 1989;14(2):159-171

17. Kearney KA, Hopkins RH, Mauss AL, Weisheit RA. Self-generated identification codes for anonymous collection of longitudinal questionnaire data. Public Opin Q. 1984;48(1B):370-378

18. Boyce W, Torsheim T, Currie C, Zambon $\mathrm{A}$. The family affluence scale as a measure of national wealth: validation of an adolescent self-report measure. Soc Indic Res. 2006;78(3):473-487

19. Krishnan-Sarin S, Morean ME, Camenga DR, Cavallo DA, Kong G. E-cigarette use among high school and middle school adolescents in Connecticut. Nicotine Tob Res. 2015;17(7):810-818
20. Wills TA, Knight R, Williams RJ, Pagano I, Sargent JD. Risk factors for exclusive e-cigarette use and dual e-cigarette use and tobacco use in adolescents. Pediatrics. 2015;135(1). Available at: www.pediatrics.org/cgi/content/full/ 135/1/e43

21. US Department of Health and Human Services. Preventing Tobacco Use Among Youth and Young Adults: A Report of the Surgeon General. Atlanta, GA: US Department of Health and Human Services, Centers for Disease Control and Prevention, Office on Smoking and Health; 2012

22. Kong G, Morean ME, Cavallo DA, Camenga DR, Krishnan-Sarin S. Reasons for electronic cigarette experimentation and discontinuation among adolescents and young adults Nicotine Tob Res. 2015;17 (7):847-854

23. Zhu SH, Sun JY, Bonnevie E, et al. Four hundred and sixty brands of e-cigarettes and counting: implications for product regulation. Tob Control. 2014;23(suppl 3):iii3-iii9

24. Ambrose BK, Day HR, Rostron B, et al. Flavored tobacco product use among US youth aged 12-17 years, 2013-2014. JAMA. 2015;314(17):1871-1873

25. Kong G, Morean ME, Cavallo D, Camenga DR, Krishnan-Sarin S. Sources of electronic cigarette acquisition among adolescents in Connecticut. Tob Regul Sci. 2017;3(1):10-16
26. Williams RS, Derrick J, RibisI KM. Electronic cigarette sales to minors via the internet. JAMA Pediatr. 2015;169(3):e1563

27. Yuan M, Cross SJ, Loughlin SE, Leslie FM. Nicotine and the adolescent brain. J Physiol. 2015;593(16):3397-3412

28. Counotte DS, Smit AB, Pattij T, Spijker $S$. Development of the motivational system during adolescence, and its sensitivity to disruption by nicotine. Dev Cogn Neurosci. 2011;1 (4):430-443

29. Farsalinos KE, Spyrou A, Tsimopoulou K, Stefopoulos C, Romagna G, Voudris $V$. Nicotine absorption from electronic cigarette use: comparison between first and new-generation devices. Sci Rep. 2014;4:4133

30. Wagener TL, Floyd EL, Stepanov I, et al. Have combustible cigarettes met their match? The nicotine delivery profiles and harmful constituent exposures of second-generation and thirdgeneration electronic cigarette users. Tob Control. 2017;26(e1):e23-e28

31. Food and Drug Administration, HHS Deeming tobacco products to be subject to the federal food, drug, and cosmetic act, as amended by the family smoking prevention and tobacco control act; restrictions on the sale and distribution of tobacco products and required warning statements for tobacco products. Final rule. Fed Regist. 2016;81 (90):28973-29106 